\title{
A REMARK ON THE INTERSECTION OF TWO LOGICS
}

\author{
SATOSHI MIURA
}

The intuitionistic logic LJ and Curry's LD (cf. [1], [2]) are logics stronger than Johansson's minimal logic LM (cf. [3]) by the axiom schemes $\wedge \rightarrow x$ and $y \vee(y \rightarrow \hat{\wedge})$, respectively. However, LM can not be taken literally as the intersection of these two logics $\mathbf{L J}$ and $\mathbf{L D}$, which is stronger than $\mathbf{L M}$ by the axiom scheme $(\curlywedge \rightarrow x) \vee y \vee(y \rightarrow \curlywedge)$. In pointing out this situation, Prof. K. Ono suggested me to investigate the general feature of the intersection of any pair of logics. In this paper, I will show that the same situation occurs in general. I wish to express my thanks to Prof. K. Ono for his kind guidance.

Let $\mathbf{A}$ be a logic having logical constants, implication $(\rightarrow)$ and disjunction $(\mathrm{V})$ (and universal quantification ( ) for predicate logics), together with all such inference rules with respect them that are admitted in the intuitionistic logic (cf. [5], p. 81). For any logic L, let us denote by $\Pi_{L}$ the class of all provable propositions in $\mathbf{L}$.

Theorem. Let $\mathrm{B}, \mathbf{C}$, and $\mathbf{D}$ be the logics formed from $\mathbf{A}$ by adjoining the axiom schemes

$$
\begin{aligned}
& \left(u_{1}\right) \cdots\left(u_{p}\right) f\left(x_{1}, \ldots, x_{s}\right), \quad(p=0,1,2, \ldots), \\
& \left(v_{1}\right) \cdots\left(v_{q}\right) g\left(y_{1}, \ldots, y_{t}\right), \quad(q=0,1,2, \ldots), \\
& \left(u_{1}\right) \cdots\left(u_{p}\right) f\left(x_{1}, \ldots, x_{s}\right) \vee\left(v_{1}\right) \cdots\left(v_{q}\right) g\left(y_{1}, \ldots, y_{t}\right),
\end{aligned}
$$

$$
(p, q=0,1,2, \ldots),
$$

respectively; where $u_{i}^{\prime}$ 's and $v_{j}^{\prime}$ 's are object variables $(p=q=0$ for proposition logics $),\left(u_{1}\right) \cdots\left(u_{p}\right) f\left(x_{1}, \ldots, x_{s}\right)$ and $\left(v_{1}\right) \cdots\left(v_{q}\right) g\left(y_{1}, \ldots, y_{t}\right)$ are expressible in $\mathbf{A}, x_{i}^{\prime}$ 's and $y_{j}^{\prime}$ 's are metalogical variables for propositions, predicates, or relations, and $s \leq t$. Then,

I.

$$
\Pi_{\mathrm{D}}=\Pi_{B} \cap \Pi_{C} .
$$

II. $\mathbf{B}$ and $\mathbf{C}$ formed from $\mathbf{D}$ by adjoining the axiom schemes

$$
(4)_{\mu}\left(w_{1}\right) \cdots\left(w_{r}\right)\left(g\left(y_{1}, \ldots, y_{t}\right) \rightarrow f\left(y_{\mu(1)}, \ldots, y_{\mu(s)}\right)\right), \quad(r=0,1,2, \ldots),
$$

Received August 19, 1965. 
$(5)_{\mu} \quad\left(w_{1}\right) \cdots\left(w_{1}\right)\left(f\left(y_{\mu(1)}, \ldots, y_{\mu(s)}\right) \rightarrow g\left(y_{1}, \ldots, y_{t}\right)\right), \quad(r=0,1,2, \ldots)$, respectively; where $w_{i}$ 's are object variables $(r=0$ for proposition logics), $1 \leq \mu(k)$ $\leq t, k=1, \ldots, s$, and $\mu(i)=\mu(j)$ implies $i=j$.

Proof of I. We prove the theorem for predicate logics. For proposition logics, we can prove it as a special case of this proof.

Let us denote $\left(x_{1}, \ldots, x_{s}\right)$ and $\left(y_{1}, \ldots, y_{t}\right)$ simply by $\boldsymbol{x}$ and $\boldsymbol{y}$, respectively. Clearly, $\Pi_{D} \subseteq \Pi_{B} \cap \Pi_{C}$. To show $\Pi_{B} \cap \Pi_{C} \subseteq \Pi_{D}$, take any proposition $\mathfrak{p}$ in $\Pi_{B} \cap \Pi_{C}$. Assume that $\mathfrak{p}$ can be proved in $\mathbf{B}$ (in $\mathbf{C}$ ) by making use of propositions of the form (1) (of the form (2)) $m$ times ( $n$ times), and let $l$ be the maximum number of $m$ and $n$. Then, propositions of the forms

$$
\begin{aligned}
& F_{m}(\mathfrak{p}) ₹ \bar{f}_{n \rightarrow} \rightarrow\left(\bar{f}_{m-1} \rightarrow\left(\cdots \rightarrow\left(\bar{f}_{1} \rightarrow \mathfrak{p}\right) \cdots\right)\right), \\
& G_{n}(\mathfrak{p}) \geqslant \bar{g}_{n} \rightarrow\left(\bar{g}_{n-1} \rightarrow\left(\cdots \rightarrow\left(\bar{g}_{1} \rightarrow \mathfrak{p}\right) \cdots\right)\right)
\end{aligned}
$$

must be provable in $\mathbf{A}$, hence in $\mathbf{D}$; where $\bar{f}_{i}$ and $\bar{g}_{j}$ are propositions of the forms $\left(u_{1}\right) \cdots\left(u_{p}\right) f\left(\boldsymbol{a}_{i}\right)$ and $\left(v_{1}\right) \cdots\left(v_{q}\right) g\left(\boldsymbol{b}_{j}\right)$, respectively. Naturally, $F_{0}(\mathfrak{p})$ as well as $G_{0}(p)$ stands for $\mathfrak{p}$. It is enough to show that any proposition of the form

$$
H_{m, n} \geqslant F_{m}(\mathfrak{p}) \rightarrow\left(G_{n}(\mathfrak{p}) \rightarrow \mathfrak{p}\right)
$$

is provable in $\mathbf{D}$ under the assumption that any propositions of the forms $H_{r, s}$ are provable in $\mathbf{D}$ for all $r, s<l$.

According to the practical way of description introduced by Ono (cf. [4], [5]), we have

Proof of $H_{m, n} / \mathbf{A}, \mathbf{B} \rightarrow \mathbf{c}$.
A) Assume $F_{m}(\mathfrak{p})$.
B) Assume $G_{n}(p)$.
c)) $\mathfrak{p} / \mathbf{c a}, \mathbf{c b}$, cc for $m>0$ and $n>0$ (c follows immediately from A for $m=0$, and from B for $n=0$.).
ca)) $\bar{f}_{m \rightarrow p} \quad /$ caA $\rightarrow$ cae. caA $)$ Assume $\bar{f}_{m}$.
cab) $F_{m-1}(\mathfrak{p}) / \mathbf{A}, \mathbf{c a A}$.
cac)) $\bar{g}_{n} \rightarrow \mathfrak{p} \quad /$ cacA $\rightarrow$ cacd. $\quad$ cac $\left.\mathbf{A}\right)$ Assume $\bar{g}_{n}$.
cacb) $G_{n-i}(\mathfrak{p}) /$ B, cacA.
cacc) $F_{m-1}(\mathfrak{p}) \rightarrow\left(G_{n-1}(\mathfrak{p}) \rightarrow \mathfrak{p}\right) \quad$ /Assumption of induction.
cacd) $\mathfrak{p}$ /cacc, cab, cacb.
cad) $\quad F_{m-1}(\mathfrak{p}) \rightarrow\left(\left(\bar{g}_{n} \rightarrow \mathfrak{p}\right) \rightarrow \mathfrak{p}\right)$ /Assumption of induction for $l>1$; tautological 
for $l=1$.

cae) $\mathfrak{p} / \mathrm{cad}$, cab, cac.

cb)) $\bar{g}_{n \rightarrow \mathfrak{p}} /$ similarly as ca.

cc) $\vec{f}_{m} \vee \bar{g}_{n} /(3)$.

Proof of II. Even in A, (1) and (2) are equivalent to "(3) and (4)" and "(3) and $(5)_{\mu}$ ", respectively.

Remark. For different permutations $\mu$ and $\mu^{\prime},(4)_{\mu}$ and $(4)_{\mu^{\prime}}\left((5)_{\mu}\right.$ and $\left.(5)_{\mu}\right)$ are mutually equivalent in D. (1) is decomposed into (3) and (4) $)_{\mu}$, and (2) into (3) and $(5)_{\mu}$. However, we can decompose (1) and (2) into still weaker components, as Fig. 1 shows. Namely, (1) is decomposed into $(9)_{\mu}$ and $(4)_{\mu}$, and (2) into $(9)_{\mu}$ and $(5)_{\mu}$, where

$(9)_{\mu}$

$$
\begin{array}{r}
\left(u_{1}\right) \cdots\left(u_{p}\right) f\left(y_{\mu(1)}, \ldots, y_{\mu(s)}\right) \vee\left(v_{1}\right) \cdots\left(v_{q}\right) g\left(y_{1}, \ldots, y_{t}\right), \\
(p, q=0,1,2, \ldots) .
\end{array}
$$

Motivated by this circumstance, it would be of some interest to seek for the weakest axiom scheme (or inference rule) under those which form $\mathbf{B}(\mathbf{C})$ by being added to $D$. However, it would be hard to find out anything of this kind, since such axiom scheme (or inference rule) must be equivalent to the metalogical assumption that the proposition scheme $\left(v_{1}\right) \cdots\left(v_{q}\right) g\left(y_{1}, \ldots, y_{t}\right)$ $\left(\left(u_{1}\right) \cdots\left(u_{p}\right) f\left(x_{1}, \ldots, x_{s}\right)\right)$ in the whole implies any proposition of the form $f\left(x_{1}, \ldots, x_{s}\right)\left(g\left(y_{1}, \ldots, y_{t}\right)\right)$.

Example 1. LJ and $\mathbf{L N}$ (named by Ono, cf. [6], [7]) are formed from $\mathbf{L M}$

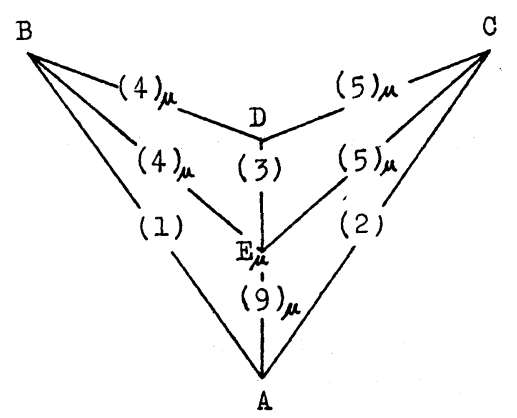

Fig. 1

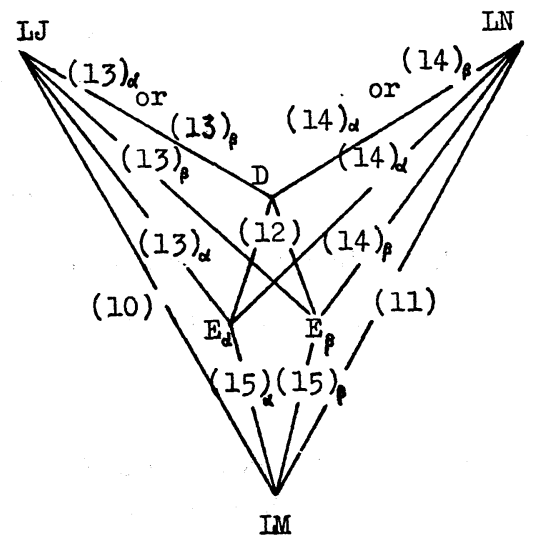

Fig. 2 


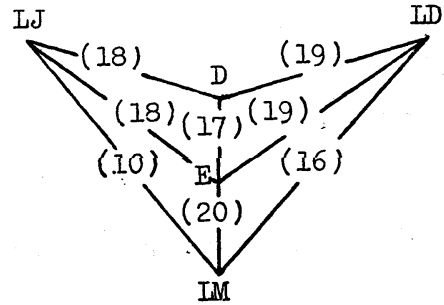

Fig. 3

by adjoining the axiom scheme

$$
y_{1} \vee\left(y_{1} \rightarrow y_{2}\right) \quad \text { (a deformation of Peirce's rule), }
$$

respectively. The mutual relation of formulas and logics is shown in Fig. 2, where

$$
\begin{gathered}
(\wedge \rightarrow x) \vee y_{1} \vee\left(y_{1} \rightarrow y_{2}\right), \\
\left(y_{1} \rightarrow y_{2}\right) \rightarrow\left(\wedge \rightarrow y_{1}\right), \\
\left(y_{1} \vee\left(y_{1} \rightarrow y_{2}\right)\right) \rightarrow\left(\wedge \rightarrow y_{2}\right), \\
\left(\wedge \rightarrow y_{1}\right) \rightarrow\left(y_{1} \vee\left(y_{1} \rightarrow y_{2}\right)\right), \\
\left(\Lambda \rightarrow y_{2}\right) \rightarrow\left(y_{1} \vee\left(y_{1} \rightarrow y_{2}\right)\right), \\
\left(\wedge \rightarrow y_{1}\right) \vee\left(y_{1} \rightarrow y_{2}\right), \\
\left(\wedge \rightarrow y_{2}\right) \vee y_{1} \vee\left(y_{1} \rightarrow y_{2}\right),
\end{gathered}
$$

Example 2. As a special case of Example 1, we have Fig. 3, where

$$
\begin{gathered}
y \vee(y \rightarrow \wedge) \quad(\mathrm{cf.}[1],[2]), \\
(\wedge \rightarrow x) \vee y \vee(y \rightarrow \wedge), \\
(y \rightarrow \wedge) \rightarrow(\wedge \rightarrow y), \\
(\text { 人 } \rightarrow y) \rightarrow(y \vee(y \rightarrow \wedge)), \\
(\wedge \rightarrow y) \vee(y \rightarrow \wedge) .
\end{gathered}
$$

To show characteristic feature as simply as possible, we have omitted object variables in describing above examples.

\section{ReferenCes}

[1] Curry, H. B.: The system LD, J. Symbolic Logic, vol. 17 (1952), pp. 35-42.

[2] Curry, H. B.: Foundations of mathematical logic, (1963), New York.

[3] Johansson, I.: Der Minimalkalkül, ein reduzierter intuitionistischer Formalismus, Compositio Mathematica, vol. 4 (1936), pp. 119-136. 
[4] Ono, K.: On a practical way of describing formal deductions, Nagoya Math. J., vol. 21 (1962), pp. 115-121.

[5] Ono, K.: A certain kind of formal theories, Nagoya Math. J., vol. 25 (1965), pp. 59-86.

[6] Ono, K.: On universal character of the primitive logic, Nagoya Math. J., vol. 27 (1966), pp. 331-353.

[7] Peirce, C. S.: On the algebra of logic-A contribution to the philosophy of notation, Amer. J. of Math., vol 7 (1885), pp. 180-202.

Toyota Technical College 